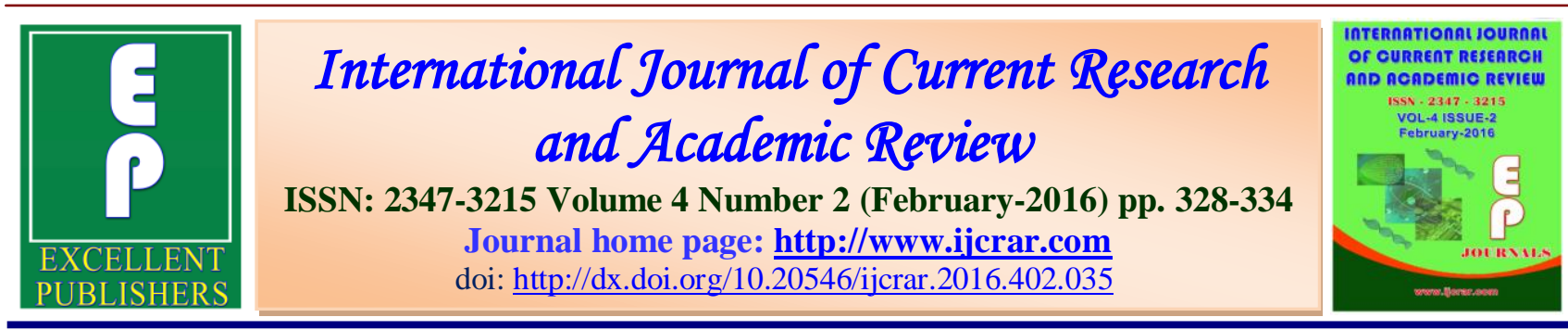

\title{
Morphological Analysis of Salivary Gland in three important Mosquito Genera (Anopheles stephensi, Culex quinquefasciatus and Aedes aegypti)
}

\section{Arti Prasad, Devendra Kumar and Azra Parveen*}

Insect microbial and Herbal Control Laboratory, Department of Zoology, University College of Science, Mohan Lal Sukhadia University, Udaipur, Rajasthan, India

*Corresponding author

KEYWORDS

Salivary Gland,

Polytene

Chromosome,

Anopheles

stephens,

Culex

quinquefasciatus

and Aedes aegypti
A B S T R A C T

The salivary gland play an important role in transmission of pathogen in mosquitoes and other vector borne diseases. In this respect in the present study morphology of Anopheles stephensi, Culex quinquefasciatus and Aedes aegypti, mosquitoes salivary gland is comparatively studied under a light microscope. The salivary gland preparation shows considerable morphological difference in adult and larvae of Anopheles stephensi as compared to Culex quinquefasciatus and Aedes aegypti, while it is almost similar with minute variation in Culex quinquefasciatus and Aedes aegypti, mosquitoes. The purpose therefore of this article is to discriminate clearly between the morphology of salivary gland in different mosquito genera and their importance.

\section{Introduction}

The family Culicidae of the order Diptera is subdivided into three subfamilies, Anophelinae, Toxorhynchitinae and Culicinae, which contains about 3400 species of mosquitoes (Service, 1993). Mosquito's are the nuisance pest, several diseases are associated to the mosquito human infection such as malaria, filariasis, yellow fever, dengue and other arboviral diseases and endemicity in India is mainly due to mosquito borne diseases. Thus these mosquito species that are vectors of human disease, particularly those belonging to the three principal genera, Aedes, Culex and Anopheles would be an urgent priority research. The key requirement for spread and infectivity of the parasite is an infected blood meal which starts parasite transmission cycle. The blood feeding is well organized biological process which involves use of anticoagulants that cause severe immune reaction by the host, and reduce the parasite load for its survival.

Concerning the spread of disease the role of salivary gland can't be neglected. The 
mosquito salivary gland plays a crucial role in food ingestion and pathogen transmission. Salivary glands of mosquitoes perform several important functions for their effective survival while carrying and transmitting pathogens and parasites. The fine structural design and physiology of the salivary gland makes it an effective organ to perform various functions including blood feeding and parasite transmission but in females only. Male mosquitoes are incapable of blood-feeding due to the absence of specialized organ for biting; consequentially they only feed on nectar. Vasodilatation is important in facilitating efficient blood feeding, and hence for transmission of pathogens and parasites. The salivary glands are sexually dimorphic and this structural and functional difference between the male and female organs shows the ability of the female to cause infection alone. (James, 1994; Stark and James, 1996).

Mosquito saliva contains anti-haemostatic agents, which help the mosquito to feed effortlessly and efficiently, avoiding the defensive response of the host (Beerntsen et al., 2000).The saliva contain certain agents which are secreted in the different lobes of the salivary gland (Ribeiro et al., 1984; James and Rossignol, 1991; James, 1994). Mosquito salivary gland is a broad area of research due to its prime involvement in vector transfer. Hence the present work is immeasurably helpful to those researchers who are working in this area. Dhar, R. and Kumar.(2003) review briefly on the role of mosquito salivary glands its biological processes and mechanisms pertinent to transmission of malaria parasite.

The study of larval salivary gland is another field of research due to the presence of polytene chromosome in it. These giant chromosomes were first described in larval chironomids by Balbiani (1881) but only accorded proper significance 50 years later by Heitz and Bauer (1933).

The polytene chromosomes of Dipteran salivary glands offer a natural system in which differential gene activity can be analysed unswervingly at the level of the genes themselves. The study on morphology and development of salivary gland and their chromosome was done by N.Rishikesh (1959). Moreira C.K, Bijovsky A.T.(1999) A literature survey showed several reports on the study of the polytene chromosomes in Culex quinquefasciatus (Berger 1937, Kitzmiller 1956, Sharma et al. 1969, Kanda 1970, Patnaik et al. 1989).

\section{Materials and Methods}

\section{Mosquito Rearing}

Separate culture of Anopheles Stephensi,Culex quinquefasciatus and Aedes aegypti, mosquitoes were reared in laboratory (Insect Microbial and herbal control laboratory, Department of Zoology, MLSU, Udaipur). Field collected larva reared to adult and identified for species and then pure culture is maintained at temperature $28 \pm 2$ and with relative humidity between $70-80 \%$ in the laboratory. The larvae fed with dog biscuits and yeast powder in the ratio 3:1. 10\% Sucrose solution and blood meal were used for adult.

\section{Mosquito Salivary Gland Dissection}

A binocular stereoscopic microscope permitting magnifications of 30-40 diameters is essential for dissection of both adult and larvae. Dissection of adult mosquito salivary gland tissues were done by following the method explained by Judy et al.2007. Mosquito's salivary gland dissection requires prior preparation of $1 \mathrm{X}$ 
Phosphate Buffered Saline (1X PBS) solution and anesthetization. The salivary glands are located at the anterior portion of the thorax and can be removed by using a fine needle. The dissected salivary glands consist of three lobes: two lateral lobes and a medial lobe.

Regarding larval dissection very few literatures are available. Although Russel et al.(1946) and Trembley,H.L. (1955) cited good literature about it.Dissecting instruments in case of larval dissection was prepared in our laboratory. The dissecting needles were made by embedding no.1 insect pins into orangewood sticks. Both bend pins as well as straight pins are essential. Temporary slide preparations were done after staining with acetocarmine and microphotographs were taken under $10 \mathrm{x}$ magnifications in both cases (adult and larvae).

\section{Results and Discussion}

\section{Adult Mosquito Salivary Gland}

After observing the prepared slides it was found that the adult mosquito's salivary glands are found in pair in the thorax and each gland has three lobes, two lateral and one median. The ducts from each lobe fuse so as to form a common salivary duct which opens at the base of the hypopharynx. Significant difference was noted when these prepared slides were compared. The duct in Culicine mosquitoes extends the length of each lobe, whereas in Anophelines, it extends only part-way along the lobe. The glands are much larger in females than in males. Comparative analysis of morphology of salivary gland of three mosquito genera shows similarity in Culex and Aedes mosquitoes in which it is long trilobed structure with costricted or wavy margins while in Anophelines the three lobes are comparatively short with smooth margin (Fig.1).

\section{Salivary Gland of Larvae}

The larval salivary glands consist of one pair of elongated bodies present in the larval thorax. The gland opens anteriorly into a long, slender duct. These ducts unite to form a common salivary duct. Each gland consists of two lobes. The small proximal lobe is spherical in shape and consists of large cells, which show giant nuclei. The larger, elongated, distal lobe is consisting of small cells with small nuclei. These nuclei enclose polytene chromosomes. Morphologically the larval salivary gland consists of two lobes proximal and distal. Considerable variation is seen in there morphology when compared among Anopheles aedes and culex mosquito. In Anopheles stephensi the proximal lobe is almost spherical but in other two mosquitoes it is irregular. Significant difference is observed in distal lobe which is found to be expanded apple shaped in Anopheles, cigar shaped in Culex and elongated shaped in Aedes (Fig. 2).

\section{Morphological Study of Anopheles stephensi Larval Salivary Gland}

The life cycle of Anopheles stephensi consists of four larval stages. When comparative analysis of salivary gland of various larval instars was done observable differences were identified. The proximal lobe of the first instar larva remains small and rounded which is almost of same size in next stages. The distal lobe, on the other hand, shows a disproportionate increase in over-all size in $2^{\text {nd }}, 3^{\text {rd }}$ and $4^{\text {th }}$ larvae owing to the accumulation of secretory matter within its lumen. The cytoplasm of the distal lobe cells is vacuolated, indicating secretory activity. The entire gland is covered by a thin membrane. Also the number of cells is 
Int.J.Curr.Res.Aca.Rev.2016; 4(2): 328-334

also increasing as we proceed from $1^{\text {st }}$ to $4^{\text {th }}$ larvae. In each cell prominent nuclei is visible in each case but clearer in $4^{\text {th }}$ instar which thus gives good preparation of polytene chromosome.

Fig.1 Microphotographs of Adult Mosquito Salivary Gland
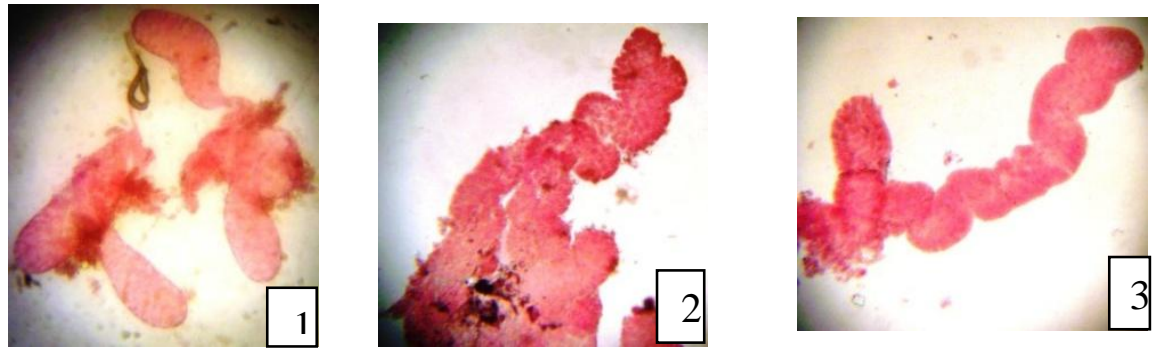

1-Salivary gland of Anopheles stephensi

2-Salivary gland of Ades aegypti

3-Salivary gland of Culex quinquefasciatus

Fig.2 Microphotographs of Larval Salivary Gland
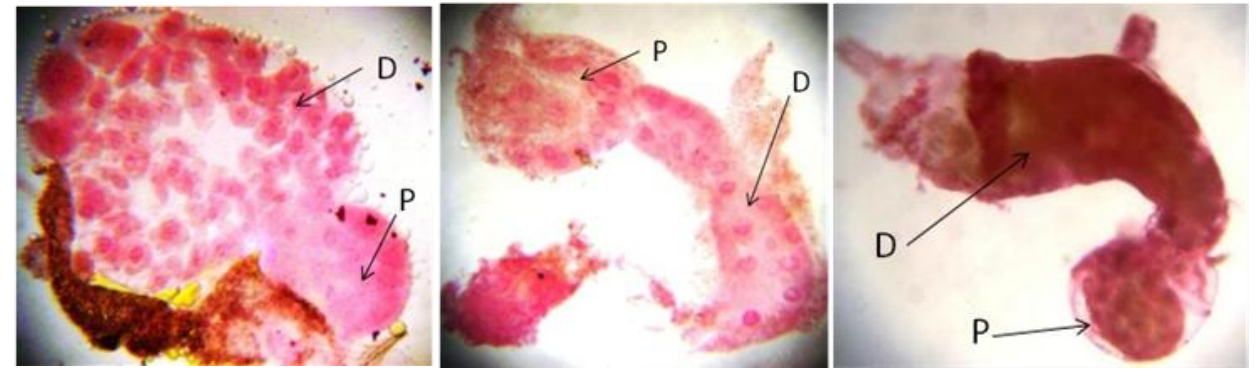

1. Anopheles stephensi

2. Aedes aegypti larvae

3. Culex quinquefasciatus

P-Proximal lobe

D-Distal lobe

Fig.3 Light Microscopic Preparation of Anopheles stephensi Larval Salivary Gland From 1st - 4th Instar

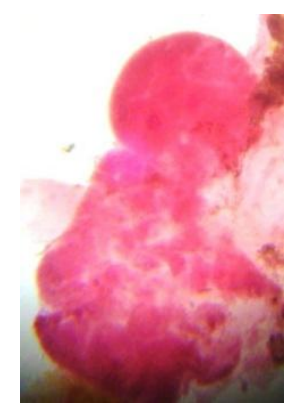

$1^{\text {st }}$

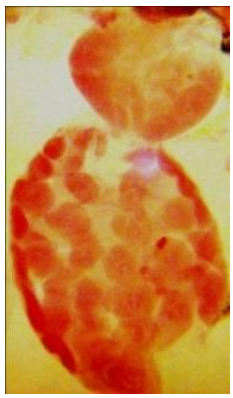

$2^{\text {nd }}$

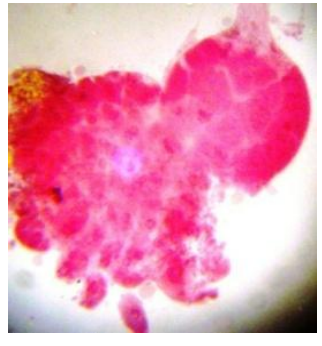

$3^{\text {rd }}$

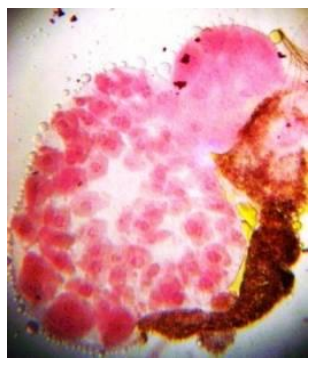

$4^{\text {th }}$ 
The present study on morphology of adult and larval mosquito salivary glands of Aedes, Culex and Anopheles species reveals similarity with the work of number of authours. Earlier works on the morphological details of mosquito salivary glands has been described for Aedes aegypti, Anopheles stephensi, Culex pipiens, Anopheles darlingi, and Culex quinquefasciatus (Orr et al., 1961; Wright, 1969; Janzen and Wright, 1971; Barrow et al., 1975; Moreira-Ferro et al., 1999; da Cunha Sais et al., 2003). As observed for Anopheles mosquitoes, the salivary glands of adult composed of three identical lobes, one short medial lobe and two longer lateral ones which are single lobed. We also observed that besides having number of lobes in common which is three in these three species, the length and morphology vary considerably in Culex and Aedes as compared to Anopheles. Our findings also show that Anopheles mosquito salivary glands are short with smooth boundary while Culex and Aedes have comparatively longer lobes with wavy or constructed margin. Morphology of mosquito salivary glands studied are similar to other mosquito species previously reported ( Moreira et al., 2001; Jariyapan et al.,2002; Siriyasatien et al., 2005; Suwan et al.,2002: Mellink et al., 1976; Poehling, 1979; Al-Ahdal et al., 1990; Marinotti et al., 1990; Andrews et al., 1997; Nascimento et al., 2000). The three lobes in salivary gland is previously reported in Anopheles Darling (Moreira et al., 1999) and Aedes aegypti (Janzen et al., 1971). Salivary glands of mosquitoes are thus important in performing several functions for effective survival of the insect and maintaining the tenacity to carry pathogens and parasites. The unique structural and physiology of the salivary gland makes it an ideal organ which helps in blood feeding and effective parasite transmission. And histological details of female salivary glands have been studied in Aedes aegypti and Aedes togoi (Beckett, 1990).

In conclusion, comparative study of morphology of mosquito salivary glands might relate to blood feeding process in these mosquitoes and disease transmission. Further study of larval salivary gland and there by polytene chromosome would provide valuable data for species identification and effectively controlling mosquito borne diseases.

\section{Acknowledgement}

The authors are thankful to management of MLSU for providing necessary facilites and team of Insect herbal control laboratory for their cooperation.

\section{References}

Al-Ahdal, M.N., Al-Hussain, K., Thorogood, R.J., Rrilly, H.C., Wilson, J.D.,1990. Protein constituents of mosquito saliva: studies on Culex molestus. J Trop Med Hyg.; 93: 98105.

Andrews, L., Laughinghouse, A., Sina, B.J.,1997. Lectin binding characteristics of male and female salivary gland proteins of Anopheles gambiae: identification and characterization of female specific glycoproteins. Insect Biochem Mol Biol.27: 159-66.

Balbiani, E. G., 1881. Sur la structure du noyau des cellules salivaires chez les larves de Chironomus. Zool. Ann. 4, 637-641; 662-666.

Barrow, P.M., McIver, S.B. \& Wright, K.A., 1975. Salivary glands of female Culex pipiens: morphological changes associated with maturation and bloodfeeding. Canadian Entomologist 107: 1153-1160. 
Beckett, E.B., 1990. Development and ageing of the salivary glands of adult female Aedes aegypti (L.) and Aedes togoi (Theobald) mosquitoes (Diptera: Culicidae). International Journal of Insect Morphology and Embryology 19: 277-290.

Beerntsen, B.T.,James.A.A. and Christensen,B.M., 2000.Genetics of mosquito vector competence, Microbiology and Molecular biology reviews64:427-433.

Berger, C. A., 1937. Additional evidence of reported chromosome division without mitotic activity. Am. Nat.71:187-190.

Da Cunha Sais, T., de Moraes, R.M., Ribolla, P.E., de Bianchi, A.G., Marinotti, O. \& Bijovsky, A.T., 2003. Morphological aspects of Culex quinquefasciatus salivary glands. Arthropod Structure and Development 32: 219-226.

Dhar,R. and Kumar,N.,2003. Role of mosquito salivary glands. Current Science, vol. 85, no. 9.

Heitz, E. and Bauer, H., 1933. Beweise fur die Chromosomennatur der Kernschleifen in den Knauelkernen bon Bibio hortulensii L. Z. Zellforsch. mikrosk. Anat. 17, 67-82.

James, A. A., 1994. Molecular and biochemical analyses of the salivary glands of vector mosquitoes. Bull. Inst. Pasteur 92,113 -150

James, A.A., Rossignol, P.A.,1991. Mosquito salivary glands: parasitological and molecular aspects. Parasitol Today. 7: 267-71.

Janzen, H.G. and Wright, K.A., 1971. The salivary glands of Aedes aegypti (L.): an electron microscope study. Canadian Journal of Zoology 49: 1343-1346.

Jariyapan, N., Harnnoi, T.,2002. Preliminary study of salivary gland proteins of the mosquito Aedes togoi (Theobald).

Chiang Mai Med Bull. 41: 21-8.

Judy, C., Jennifer, J. and Anthony, A. J.,2007. Dissection of Midgut and Salivary Glands from Ae. aegypti Mosquitoes J Vis Exp. (5): 228.

Kanda, T.,1970. The salivary gland chromosomes of Culex pipiensfalrgansWiedemann. Jpn. J. Exp. Med. 40:335-345

Kitzmiller, J. B., 1956. Salivary gland chromosomes in the Culex pipiensmolestus-fatigdr4s complex. Proc. Int. Congr. Genet. 9:674-677.

Marinotti, O., James, A.A., Ribeiro, J.M.C.,1990. Diet and salivation in female Aedes aegypti mosquitos. J Insect Physiol 36: 545-8.

Mellink, J.J., van Zeben, M.S.,1976. Age related difference of saliva composition in Aedes aegypti. Mosq News. 36: 247-50.

Moreira, C.K., Bijovsky, A.T.,1999. Morphological and biochemical analyses of the salivary glands of the malaria vector, Anopheles darlingi. Tissue and Cell. 31: 264-73.

Moreira, C.K., Marrelli, M.T., Lima, S.P and Marinotti, O.,2001. Analysis of salivary gland proteins of the mosquito Anopheles darlingi (Diptera: Culicidae). J Med Entomol. 38: 763-7.

Moreira-Ferro, C.K., Marinotti, O. and Bijovsky, A.T., 1999. Morphological and biochemical analyses of the salivary glands of the malaria vector, Anopheles darlingi. Tissue and Cell 31: 264-273.

Nascimento, E.P., Malafronte, R. Dos, S.and Marinotti, O.,2000. Salivary gland proteins of the mosquito Culex quinquefasciatus. Arch Insect Biochem Physiol. 43: 9-15.

Orr, C.W.M., Hudson, A. and West, A.S., 1961. The salivary glands of Aedes aegypti, histological-histochemical 
studies. Canadian Journal of Zoology. 39: 265- 272.

Patnaik, S., R. K. Verma, R. Prasad and C. C. Das., 1989. Banding pattern morphology in the polytene chromosome from salivary gland and malpighian tubule nuclei of Culex quinquefasciatu.r (Culicidae). Perspec. Cytol. Gene. 6:363-369.

Poehling, H.M.,1979. Distribution of specific proteins in the salivary gland lobes of Culicidae and their relation to age and blood sucking. $\mathbf{J}$ Insect Physiol. 25: 3-8.

Ribeiro, J.M., Sarkis, J.J., Rossignol, P.A., Spielman, A.,1984. Salivary apyrase of Aedes aegypti: characterization and secretory fate. Comp Biochem Physiol. 79B: 81-6.

Rishikes,N.,1959.Morphology and development of the salivary glands and their chromosomes in the larvae of Anopheles stephensi sensu strict. Bull. Org. mond. Sante Bull,Wld Hith Org. 20, 47-61.

Russel,P.F.,andWest,L.S., and Manwell,R.D.1946.Practical

Malariology, Philadephia,pp.245-246.

Service, M.W., 1993. Mosquitoes (Culicidae). In Medical insects and arachnids (ed. R. P. Lane and R. W.

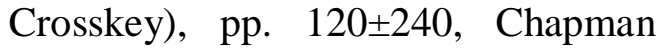
and Hall, New York.
Sharma, G. P., R. Parshad, S. L. Narang and P. Kaur. 1969. Salivary gland chromosomes of Culex pipiens fatigans. Res. Bull. Punjab Univ. 2O:541-546.

Siriyasatien, P., Tangthongchaiwiriya, K., Jariyapan, N., Kaewsaitiam, S., Poovorawan, Y., Thavara, U.,2005. Analysis of salivary gland proteins of the mosquito Armigeres subalbatus.

Southeast Asian J Trop Med Public Health. 36: 64-7.

Stark, K. and James, A. A., 1996. The salivary glands of disease vectors. In The Biology of Disease Vectors (ed. W. C. Marquardt and B. Beaty), pp.333 -348.

Suwan, N., Wilkinson, M.C., Crampton, J.M., Bates, P.A.,2002. Expression of D7 and D7-related proteins in the salivary glands of the human malaria mosquito, Anopheles stephensi. Insect Mol Biol. 11: 223-32.

Trembley,H.L.,1955.Mosquito culture techniques and experimental procedures,Amer.Mosq.Cont.Assoc.B ull.No.3,pp.15-16.

Wright, K.A., 1969. The anatomy of salivary glands of Anopheles stephensi Liston. Canadian Journal of Zoology 47: 579- 587.

\section{How to cite this article:}

Arti Prasad, Devendra Kumar and Azra Parveen. 2016. Morphological Analysis of Salivary Gland in three important Mosquito Genera (Anopheles stephensi,Culex quinquefasciatus and Aedes aegypti). Int.J.Curr.Res.Aca.Rev. 4(2): 328-334. doi: http://dx.doi.org/10.20546/ijcrar.2016.402.035 